\title{
ERROR CONCEALMENT VIA 3-MODE TENSOR APPROXIMATION
}

\author{
Dzung Nguyen, Minh Dao, Trac Tran \\ Johns Hopkins University, \\ Dept. of Electrical and Computer Engineering, \\ Baltimore, USA \\ \{dzungnguyen, minh.dao, trac $\} @$ jhu.edu
}

\begin{abstract}
We propose a novel method for video error concealment, which is essentially a combination of non-local grouping of image patches and low-rank tensor approximation. The proposed method, though does not require the knowledge of Motion Vectors (MVs) as in traditional video concealment techniques such as Boundary Matching Algorithm (BMA) and its derivations, gives striking results in restoration of sequences with high error rate without the key frames. The method can also be customized to work on single images, for example in image inpainting tasks.
\end{abstract}

Index Terms - Error concealment, inpainting, tensor, low-rank approximation, BMA

\section{INTRODUCTION}

Data recovery is part of any modern image/video communication system. Visual data encoders working in block-based fashion, hence any lost in data transmission or damage in storage media results in corruptions of blocks or group-of-blocks, which need to be restored. Numerous techniques had been proposed for data restoration. Different methods require different involvement of the source coder, the sender/receiver, the network or combinations of those entities [1]. Similar to recent works in video concealment [2, 3, 4], in this paper we focus on developing a source coder-independent receiverbased technique, which means we only use information available at the receiver to recover lost data. This can also be seen as post-processing technique that can be used to recover leftover errors which schemes failed to deal with.

Video coders often use block motion compensation technique to eliminate redundancy in video sequences. The actual information sent to the decoder are Motion Vectors (MVs) and corrensponding block residues. Therefore it is natural to exploit MVs for concealment. At an erroneous block (that loses its MV), the Boundary Matching Algorithm (BMA) [2] try to recover the MV by selecting a best candidate from the set of neighbouring MVs and the zero MV, in terms of minimum total variation of pixels values at the block's boundary. Variations of BMA [4, 3] either focus on finding a better MV or developing more sophisticated boundary matching criteria. Such methods have the advantage of fairly low complexity, but on the other hand restrict themselves to one MV and only one reference frame in the search for best recovering data.

We propose an error concealment method that does not rely on recovering $\mathrm{MV}$, therefore are also liberated from one reference frame limitation as well as the Macro-Block (MB) grid decided by the encoder. We are free to redefine the block size, the grid and its displacement in each corrupted frame. The number of reference frames is also a free parameter. Infact, more reference frames give better recovery results and this number can be increased as much as the computational capacity allows. In a nutshell, the proposed method groups image patches in the corrupted frame with similar patches searched from the entire reference frames to form 3-mode tensors with missing data. This approach is inspired by the success of denoising methods such as non-local mean or 3-D transform-domain collaborative filtering $[5,6]$. Those tensors are then approximated using an algorithm derived from tensor n-rank (Tucker) decomposition [7] to exploit the low-rank nature in the 3rd dimension.

There is also an initial work on visual data restoration from tensor completion view point [8]. In that paper, the authors follow a different path by relying on tensor canonical rank, rather than the n-rank. The paper shows some impressive preliminary results on image/video restoration, but it implicitly assumes that data must have global low rank structure. In general, one can not make such assumption about natural images and video sequence. In our work, we explicitly show how to form tensors with certain low-rank structure from patches of visual data, and exploit that structure in our proposed tensor approximation algorithm customized for this type of tensors.

The paper is organized as follows: the next section explains the key elements of our proposal; section 3 shows pseudo code of the algorithm; section 4 presents some experimental results in video concealment and image inpainting; section 5 concludes and discusses some ideas for future work. 


\section{TENSOR CONSTRUCTION AND CONCEALMENT}

Before going into details, let us adopt some important notations in tensor algebra from [7].

An $\mathrm{N}^{\text {th }}$-order (or $\mathrm{N}$-mode) tensor $\mathcal{X}$ (in calligraphic letter) is an $\mathrm{N}$-dimensional array $\mathcal{X} \in \mathbb{R}^{I_{1} \times I_{2} \times \ldots \times I_{N}}$. Unfolded matrix of $\mathcal{X}$ along the $\mathrm{n}^{\text {th }}$ mode is denoted $\boldsymbol{X}_{(n)}$. A tensor can be decomposed to a sum of rank-1 tensors (canonical decomposition) or a product of a core tensor with matrices corresponding to its modes (Tucker decomposition). In the later form, a tensor is represented as

$$
\boldsymbol{X}=\mathcal{G} \times{ }_{1} \boldsymbol{A}^{(1)} \times{ }_{2} \boldsymbol{A}^{(2)} \ldots \times{ }_{N} \boldsymbol{A}^{(N)}
$$

where $\mathcal{G} \in \mathbb{R}^{R_{1} \times R_{2} \times \ldots R_{N}}$ is the core tensor, and $\boldsymbol{A}^{(n)} \in$ $\mathbb{R}^{I_{n} \times R_{n}}$ is a matrix whose columns are eigen vectors in mode-n. The mode-n product (operator $\times_{n}$ ), between a tensor $\mathcal{X}$ and a matrix $\boldsymbol{U} \in \mathbb{R}^{R_{n} \times I_{n}}$ is defined elementwise as $\left(\mathcal{X} \times{ }_{n} \boldsymbol{U}\right)_{i_{1} \ldots i_{n-1} j i_{n+1} \ldots i_{N}}=\sum_{i_{n}=1}^{I_{n}} x_{i_{1} \ldots i_{n} \ldots i_{N}} u_{j i_{n}}$

\subsection{Block Matching for Tensor Construction}

We slice each corrupted frame using a new MB grid, which is shifted half a block size in each spatial dimension. Each missing MB is hence divided into 4 quarters (subblocks), and all missing subblocks are put in queue. The order in which subblocks are concealed is important. A smart queueing mechanism is implemented where missing subblocks with more clean neighbors and closer to the missing boundary are prioritized. As a result, subblocks in missing area are processed from its boundary towards the center.

Once a missing subblock is selected, an image patch (MB $\boldsymbol{P}^{0}$ ) of size $N \times N$ is formed that contains the missing quarter and its clean/concealed neighbors (Figure 1). This MB is used to search for similar MBs $\left(\boldsymbol{P}^{i}\right)$ in the entire stock of $R$ reference frames. Figure 2 illustrates the grouping process. Various criteria of MB similarity had been consider in [5, 6]. We chose Block Matching criteria because of its low computational complexity.

$$
S_{i}=\left\|\boldsymbol{P}_{\Omega}^{0}-\boldsymbol{P}_{\Omega}^{i}\right\|_{1}
$$

where $\Omega$ are the indices of clean/recovered pixels in $\boldsymbol{P}^{0}$ (we will use $\bar{\Omega}$ later for indices of missing pixels).

$\boldsymbol{P}^{0}$ and several MBs with best matching scores $S_{i}$ are grouped into a 3 -mode tensor $\mathcal{X} \in \mathbb{R}^{N \times N \times K}$ where $\boldsymbol{P}^{0}$ is on top. Those $\boldsymbol{P}^{i}$ should be scaled to have the same $l_{2}$ norm as $\boldsymbol{P}^{0}$ (at indices $\Omega$ ). A good practical choise of $K$ is $K=R+1$ (if we assume one good match is found in each reference frame).

\subsection{Tensor n-rank Approximation}

Ideally, $\mathcal{X}$ is formed by $\mathrm{K}$ identical patches, therefore

$$
\mathcal{X}=\mathcal{X}_{l}+\mathcal{E}=\boldsymbol{P} \times{ }_{3} 1+\mathcal{E}
$$

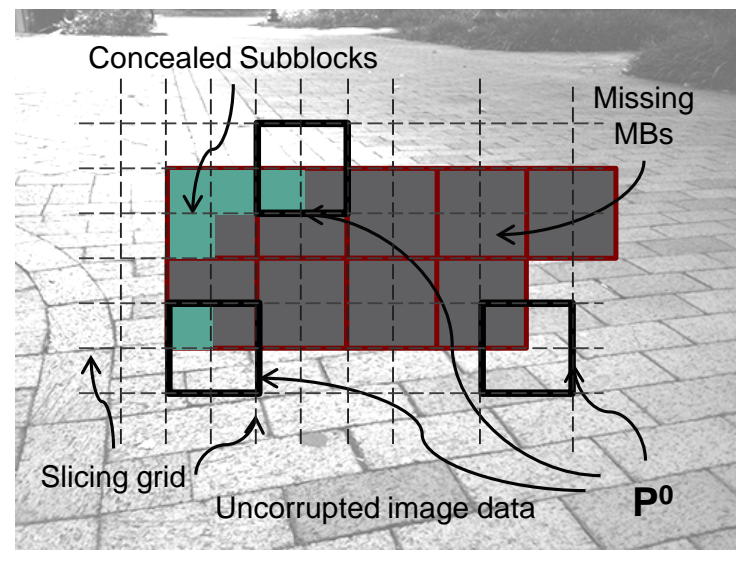

Fig. 1. New MB grid in corrupted frame and $\boldsymbol{P}^{0}$ selection

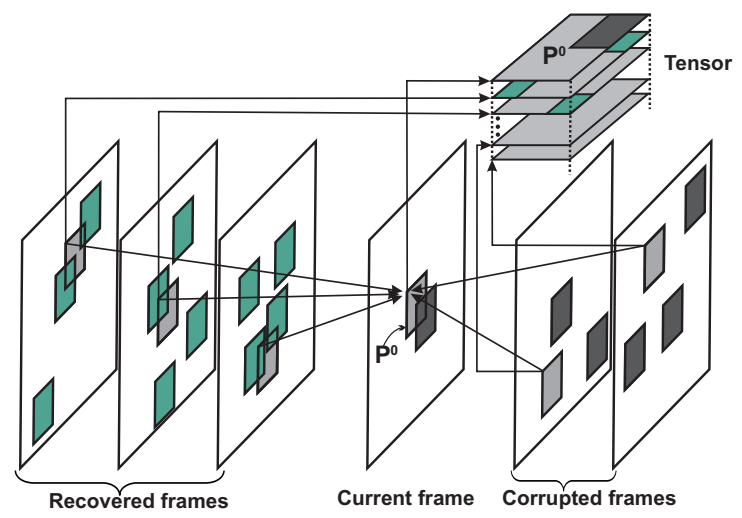

Fig. 2. Tensor build by grouping similiar MBs

where $\mathcal{X}_{l}$ has mode-3 rank equals 1 (and hence can be represented as an outer product between an image patch and a constant vector $\mathbf{1}$ ), and $\mathcal{E}$ is an error tensor (which is in general sparse). If $\mathcal{X}_{l}$ is factorized using Tucker decomposition

$$
\mathcal{X}_{l}=\mathcal{G} \times{ }_{1} A^{(1)} \times{ }_{2} A^{(2)} \times{ }_{3} A^{(3)}
$$

then we can equate (3) and (4) to have

$$
\boldsymbol{P}=\mathcal{G} \times{ }_{1} \boldsymbol{A}^{(1)} \times{ }_{2} \boldsymbol{A}^{(2)} \text { and } \boldsymbol{A}^{(3)}=\mathbf{1}
$$

In practice, patches in $\mathcal{X}$ (being grouped from different frames) are slightly different, or the actual number of identical patches may be less than $K$. Therefore, $\mathcal{X}_{l}$ may have mode- 3 rank larger than 1, but it's still a low-rank structure.

These observations suggest us to use n-rank decomposition technique to approximate $\mathcal{X}_{l}$. We base on the wellknown High-Order SVD (HOSVD) [9] while enforcing the most important eigen vector in mode- 3 to the constant vector 1 (normalized to unit $l_{2}$-norm) in each HOSVD's iteration.

\section{THE ALGORITHM}

After the tensor $\mathcal{X}$ is formed, the missing area $\left(\boldsymbol{P}^{0}\right)_{\bar{\Omega}}$ is firstly filled with the mean area from similar patches. This 
$\overline{\text { Algorithm } 1 \text { Concealment of an image patch w/ missing data }}$ 1. Form $\mathcal{X}$ from $\boldsymbol{P}^{0, \ldots, K-1}$ using Block Matching criteria

2. $(\mathcal{X}(:,:, 1))_{\bar{\Omega}}=\left(\frac{1}{K-1} \sum_{i=1}^{K-1} \boldsymbol{P}^{i}\right)_{\bar{\Omega}}$

3. Choose mode ranks $\left\{\mathrm{R}_{1}, \mathrm{R}_{2}, \mathbf{R}_{3}\right\}$, tolerance $\sigma$; Initialize $\boldsymbol{A}^{(1)}, \boldsymbol{A}^{(2)}, \boldsymbol{A}^{(3)}$

4. $\boldsymbol{A}^{(3)}(:, 1)=[1, \ldots, 1]^{T} / K$

5. for $n=1,2,3$

$$
\mathcal{Y}=\mathcal{X} \times_{1} \ldots \times_{n-1} A^{(n-1) T} \times_{n+1} A^{(n+1) T} \ldots
$$

$\boldsymbol{Y}_{n} \leftarrow$ unfold $\mathcal{Y}$ in mode $n$

$\boldsymbol{A}^{(n)} \leftarrow$ first $R_{n}$ principal component of $\boldsymbol{Y}_{n}$ end

6. $\mathcal{G}=\mathcal{X} \times{ }_{1} \boldsymbol{A}^{(1) T} \times{ }_{2} \boldsymbol{A}^{(2) T} \times{ }_{3} \boldsymbol{A}^{(3) T}$

7. $\mathcal{X}_{l}=\mathcal{G} \times{ }_{1} A^{(1)} \times{ }_{2} A^{(2)} \times{ }_{3} A^{(3)}$

8. If $\left\|\mathcal{X}_{l}-\mathcal{X}\right\|_{F} \leq \sigma$ STOP, otherwise return to Step 4.

9. Recover missing area in $\boldsymbol{P}^{0}:\left(\boldsymbol{P}^{0}\right)_{\bar{\Omega}}=\left(\mathcal{X}_{l}(:,:, 1)\right)_{\bar{\Omega}}$

is in fact a good starting estimate of the original data and help reduce the energy of the noise $\mathcal{E}$ significantly as compared to approximating $\mathcal{X}$ directly with a zero (missing) subblock in its $\boldsymbol{P}^{0}$ patch.

The algorithm then finds a best rank- $\left(R_{1}, R_{2}, R_{3}\right)$ approximation $\mathcal{X}_{l}$ of $\mathcal{X}$ in a process call Alternating Least Square (ALS) [7, 9]. In short, this is an iterative process that tries to solve for one subset of free parameters $\left(\boldsymbol{A}^{(i)} \mathrm{i}=1,2,3\right.$ or $\left.\mathcal{G}\right)$ at a time while the others being fixed. At each iteration, the first column of $\boldsymbol{A}^{(3)}$ is enforced to the constant norm-1 vector to guide the optimization to converge to the desired low-rank estimate. Since we only assume mode- 3 rank of $\mathcal{X}_{l}$ is low, $R_{3}$ is chosen small ( 3 in practice), while $\left(R_{1}, R_{2}\right)$ can be as large as $N$. Algorithm 1 elaborates all the steps.

\section{EXPERIMENTS}

We show 2 experiments on video error concealment. In the first one, the Bus CIF sequence is corrupted by random blockmissing at $15 \%$ error rate. The second one deals with missing slices in a single frame of Foreman CIF sequence, with error rate 50\%. Both are compared with standard BMA method. An experiment on object removal is setup to show our approach is also applicable to image inpainting, not only to video concealment.

\subsection{Corrupted sequence without key frames}

Experiment is performed on the first 100 frames of Bus CIF sequence. All frames are quantized in the DCT domain with step size equals 8 , therefore uncorrupted frames at the decoder would have the average PSNR of 42dB. Both BMA and our method are implemented in Matlab. At each frame, MVs refer to previous frame and BMA is allowed to use previously recovered frame as reference. Our method use 5 nearest previously recovered frames and 5 corrupted future frames in ten- sor building step. MB size (and missing block size) is $8 \times 8$. Overall, our method show a $2.47 \mathrm{~dB}$ PSNR improvement over BMA on average. The PSNR curve is shown in Fig. 3(e), while Fig. 3(a,b,c,d) shows the visual results at frame \#21. The red ovals indicate areas where BMA's failures are obviously visible.

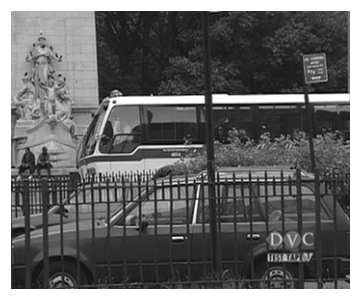

(a) Original frame

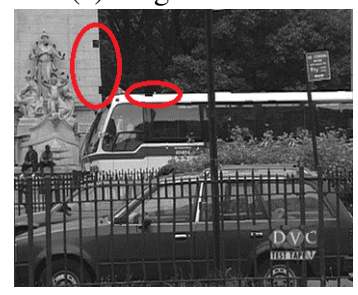

(c) BMA

PSNR $=29.19 \mathrm{~dB}$

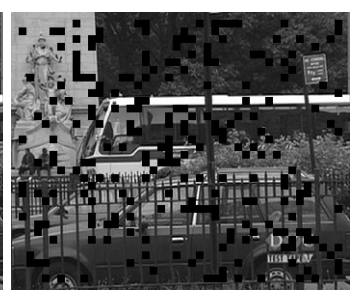

(b) Corrupted frame

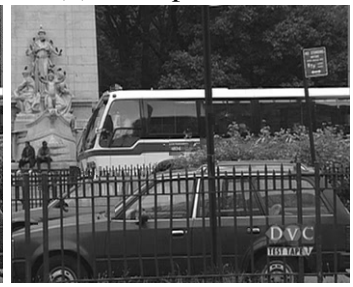

(d) Tensor approx. PSNR $=33.05 \mathrm{~dB}$

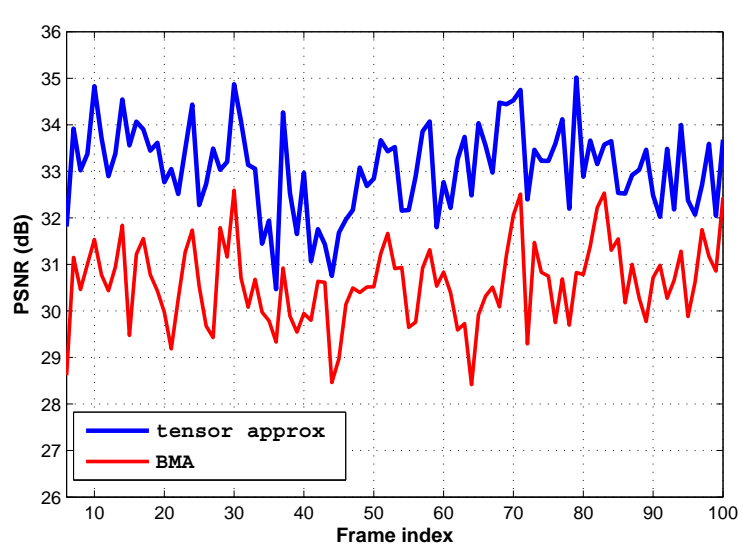

(e) PSNR comparison

Fig. 3. Recovery of Bus CIF sequence, with $15 \%$ random block missing in every frame, no key frame exists

\subsection{High error rate in an individual frame}

This experiment attack the slice-missing situation. A frame is divided into slices (each slice contains $18 \mathrm{MBs}$ ). $50 \%$ number of slices are missing at random. BMA is using previously reconstructed frame as reference, while tensor approximation method uses 2 reconstructed frames before and 2 corrupted frames after for tensor building. Blocksize is $16 \times 16$. The restoration visual quality and PSNRs are shown in Fig. 4. 


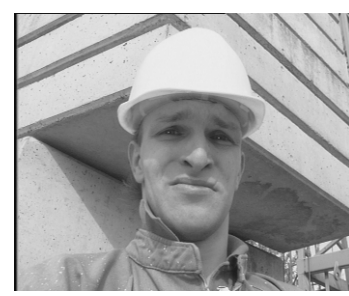

(a) Original frame

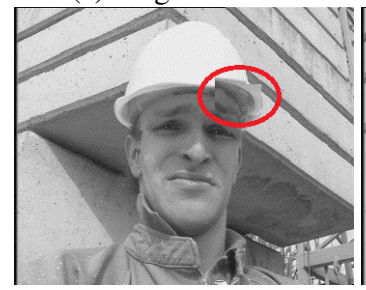

(c) BMA

$\mathrm{PSNR}=24.68 \mathrm{~dB}$

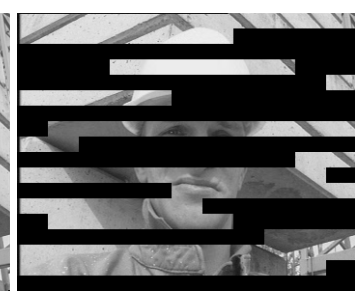

(b) Corrupted frame

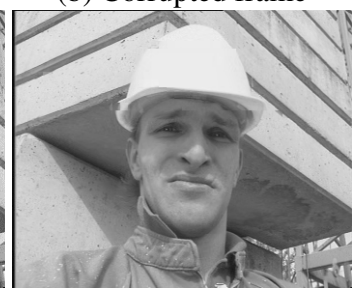

(d) Tensor approx.

$\mathrm{PSNR}=33.24 \mathrm{~dB}$
Fig. 4. Recovery of frame \#37 in Foreman CIF sequence, with $50 \%$ random slice missing

\subsection{Image inpainting}

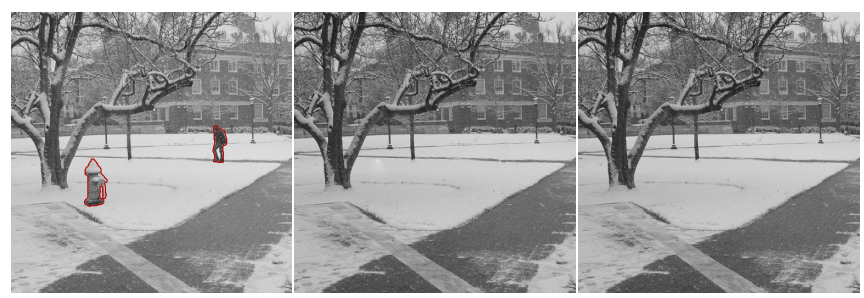

(a) Objects selection (b) Healing Brush tool (c) Tensor approx.

Fig. 5. Object removal experiment.

In this inpainting experiment, the objects (in red contours in Fig. 5(a)) are selected in Adobe's Photoshop. These selections are feeded as 'missing' maps to our algorithm. The image itself is used as the only reference frame for tensor building. The image is sliced using a $8 \times 8$ grid.

The result is compared with ouput from Healing Brush Tool in Photoshop CS4 (which is supposed to use fairly modern technology).There is no ground-truth for objective PSNR calculation, but our result is visually very competitive.

\section{FUTURE WORK}

In this paper, we propose a new successful method for error concealment/inpainting using tensor approximation. There are several perspectives that we plan to explore in near future work to extend our results.

Firstly, the issues of how to select the best block size (according to frame resolution) or how to build the best wrapping MB around missing area should be investigated. More robust tensor approximation technique will be developed to exploit the sparse nature of the error.

To contrast our technique with BMA and its derivations, in our experiment we ignore MVs and any smooth boundary constraints. Adopting those methods as an initializing stage will obviously improve our performance.

We will also perform experiments on a broader range of data and compare with other state-of-the-art techniques to have a better evaluation of our technique.

\section{REFERENCES}

[1] B.W. Wah, X. Su, and D. Lin, "A survey of errorconcealment schemes for real-time audio and video transmissions over the internet," in Multimedia Software Engineering, Proc. Int. Symposium on, 2000, pp. 17-24.

[2] Y. Wang, M.M. Hannuksela, V. Varsa, A. Hourunranta, and M. Gabbouj, "The error concealment feature in the H.26L test model," in Image Processing, Proc. Int. Conf. on, 2002, vol. 2, pp. 729-732.

[3] Y. Chen, Y. Hu, O.C. Au, H. Li, and C.W. Chen, "Video error concealment using Spatio-Temporal boundary matching and partial differential equation," Multimedia, IEEE Trans. on, vol. 10, no. 1, pp. 2-15, 2008.

[4] W. Lie and Z. Gao, "Video error concealment by integrating greedy suboptimization and kalman filtering techniques," Circuits and Systems for Video Technology, IEEE Trans. on, vol. 16, no. 8, pp. 982-992, 2006.

[5] A. Buades, B. Coll, and J.M. Morel, "A review of image denoising algorithms, with a new one," Multiscale Modeling \& Simulation, vol. 4, no. 2, pp. 490-530, Jan. 2005.

[6] K. Dabov, A. Foi, V. Katkovnik, and K. Egiazarian, "Image denoising by sparse 3-D Transform-Domain collaborative filtering," Image Processing, IEEE Trans. on, vol. 16, no. 8, pp. 2080-2095, 2007.

[7] T. G. Kolda and B.W. Bader, "Tensor decompositions and applications," SIAM Review, vol. 51, no. 3, pp. 455-500, Sept. 2009.

[8] J. Liu, P. Musialski, P. Wonka, and J. Ye, "Tensor completion for estimating missing values in visual data," in Computer Vision, IEEE 12th Int. Conf. on, 2009, pp. 21142121.

[9] Lieven De Lathauwer, Bart De Moor, and Joos Vandewalle, "A multilinear singular value decomposition," SIAM Journal on Matrix Analysis and Applications, vol. 21, Mar. 2000. 